\title{
“Mas Spec Pen”, The New Future For Cancer Diagnosis: Mini Review
}

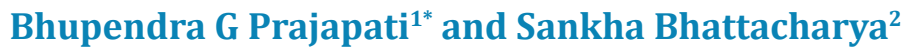 \\ ${ }^{1}$ Department of Pharmaceutical Technology, Ganpat University, India \\ ${ }^{2}$ Department of Pharmaceutics, Rofel Shri GM Bilakhia College of Pharmacy, India
}

Received: September 14, 2017; Published: September 27, 2017

*Corresponding author: Bhupendra G Prajapati, Associate Professor, Department of Pharmaceutical Technology, Ganpat University, Kherva, Mehsana, Gujarat-384012, India

\begin{abstract}
Our intention is to give an outline and aware about recent innovation called Mas-Spec Pen developed at the University of Texas. This innovation could revolutionize the cancer diagnosis in upcoming days. Jialing Zihanget al. developed this biocompatible and automated device for non-destructive diagnosis of cancerous cells. This device attached with one handheld mass spectrometer. They named it as MasSpec Pen. This device could deliver discrete water to diseased tissue to extract biomolecules for cancer diagnosis. They have analyzed this instrument applicability by using it in 253 human patient tissue samples from lung, thyroids, and ovary. With this method, it has become very easy to estimate the potential cancer biomarkers in a form of lipids, metabolites, and proteins. This device could produce $96.3 \%$ accuracy in cancer prediction. In histological composition, this device could easily identify cancerous cells within normal cells. This device could also be useful in the diagnosis of residual cancerous cells during open surgery. Nevertheless, this device can be a future for ex vivo and in vivo cancer diagnosis. Finally, we have shown how international media houses responded on this current discovery.
\end{abstract}

Keywords : MasSpec Pen; Histopathology; Breast Cancer; Lung Cancer; Mass Spectrometry

Abbreviations : PCA: Principal Component Analysis; PDMS: Polydimethylsiloxane; PE: Glycerophosphoetanolamide; PS: Glycerophosphoserines; PI: Glycerophosphoinositol; CL: Cardiolipins

\section{Introduction}

On 6th September 2017, Jialing Zihang an assistant professor, department of Chemistry, University of Texas at Austin and his center-dedicated team published a revolutionized work in science translational medicine research journal [1]. Their work could entirely eradicate cancer from its grass root. The research was comprised of performing a non-destructive tissue analysis in out of the body and diagnose of carcinoma within the living cells using a "MasSpec Pen". This "MasSpec Pen" is actually a probe, which is connected to a Mass spectrometer (Figure 1). Modern oncologists and scientist are always facing tremendous challenging's in tissues assessment and diagnosis in cancer treatment because of screening cancerous cells from normal cells. Many women diagnosed with breast cancer would undergo conserving surgery. This means the cancer surgeon has to face challenges to separate cancerous cells from within normal cells to maintain a total integrity of the mammary gland. In similar fashion, in lung cancer, complete resection of a primary tumor is the prerequisite because after surgery it was often witnessed the residual cancerous cells progress retrievable. Likewise, in ovarian cancer, the residual carcinoma cells could generate negative responses to chemotherapeutic treatment. Thus, it has become necessary to estimate negative margin assessment and excision of a complete tumour.

Moreover, interpretations of the cancer cell are a tedious process, it is a big concerned for any histopathologist to detect ontogenesis using normal frozen section method and with intraoperative frozen section method because of its time consumptions, interactions of freezing arti-facts with tissue morphology and patient life risk due to extended anaesthesia. If positive marginal oncogenic cellswere found, the patients were subjected to additional surgery, hyperactive anxiety and discomfort [2-5]. There for it is becoming an indispensable need to analysis oncogenes in molecular labels by incorporating cancer specific biomarkers to improve cancer identification and screening. At present era, distinguished ex-vivo and in-vivo tests are available using molecular imaging technology, which has very effective implications in pre-clinical and clinical studies. Some other effective techniques in cancer diagnosis are targeting biomarkers, genes sequencing technology, fluorescence probes targeting tumor cells, stimulated Raman scattering microscopy and spectroscopy, and Mass spectrometry that is used to detect molecular imaging of cancerous tissues. 


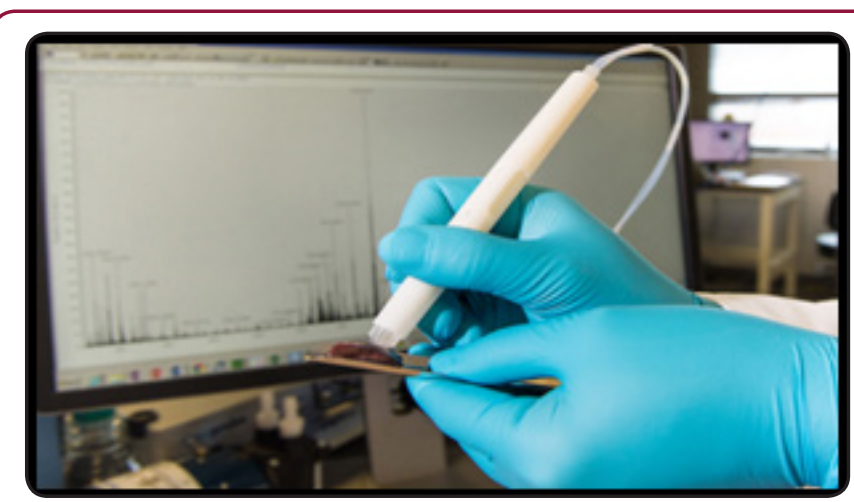

Figure 1: The MasSpec Pen while in operation.

Nevertheless, in recent time, mass analyzers are vividly using as cancer detecting tools for its accurate molecular label interpretation. Jialing Zihang and his team dedicatedly worked on improving mass analyzer's effectiveness by redefining and modifying its tool. Currently used Desorption electrospray ionization MS Imaging has certain limitations like, it cannot be used in fresh tissues and invivo analyses. The biggest drawback of this model is its dependency on tissue damage to produce molecular ions, which could be constrained \& painful for patients. To circumvent all the associated problems with the molecular oncological analysis. Jialing Zihang and his team developed a new device, which is connected to a mass analyzer. They named this device as "MasSpec Pen".

The main advantages of this pen are its atomization and biocompatibility with disposable nature. They have used this MasSpec Pen for ex-vivo molecular evaluation in human normal and cancerous cells for 253 patients. The data generated from mass analyzers comprising of diagnostic metabolized, proteins and lipids. Using selection operator and statistical technique like multivariable analysis as especially Principal Component Analysis (PCA) it has become easy to study in molecular labels so perfectly. They also demonstrated their work with one mouse model, which is best, suited for diagnosis of breast cancer during the gestation period [6-11].

\section{Operative Specifications of MasSpec Pen [1]}

The MasSpec Pen device comprising a syringe pump, which is programmable to deliver nearly 4 to $10 \mu$ l of water, a polytetrafluoroethylene tubing conduits, a handheld pen sized probe for instant sampling from biological origin. This probe is helping out excruciate tissue samples using a discrete water droplet. The polydimethylsiloxane (PDMS) tip is in three dimensions. This pen has three orifices, the first part is for delivery of a single water droplet, the second part is for delivering inert gases like nitrogen and carbon dioxide, and the third part is for collecting tissue samples by dissolving water to a mass analyzer. At the probe tips, all the orifice was interconnected with each other. There is also a vacuum system which helps in transporting extracted biomolecules. The orbit rap usually connected to the Orbit rap mass spectrometer for ionization and mass analysis. The diameter of the probe orifice reservoir at the probe tip indicates an amount of volume exposed to targeting cancerous tissue. They also claimed that at $2.77 \mathrm{~mm}$ reservoir system $10 \mu \mathrm{L}$ could connect with a tissue sample. Using a foot pedal, the entire process becomes so robust, previously within 10 seconds samples were collected by probe tip and circulated to the mass spectrometer.After use; it is advisable to clean MasSpec Pen through a rapid and automated cleaning flush or replacing the disposable tip.

They also performed molecular analysis by taking human and mouse tissue samples. In mouse sample taking $16 \mu \mathrm{m}$ tissue section from gray matters, white matters could analyses lipid contents. In mass analyzers, the samples produce negative ions, which actually alter the mass/charge ratio. The negative ion spectrum obtained from gray matter region produces vital molecular information about protonation of lipids. In this technique clearly, sphingolipids, fatty acids, Glycerophosphoetanolamide (PE), Glycerophosphoserines (PS), Glycerophosphoinositol (PI), Glycerophosphoserines (PS) and double charged cardiolipins (CL) can be analyzed.

They also tested the capacity of the MasSpec Pen by using it in human papillary thyroid carcinoma; follicular thyroid adenoma. The procedures were almost same. It was observed that nonmalignant cells often comprise of soft connected tissues such as stroma provide less abundant mass spectra with comparison with cancerous cells of breast and lung cancer cells. The fundament achievements of this technique are no characteristic damage on the surface after MasSpec Pen analysis. Zhang et al. analysis of cancer carried away with estimating 253 human tissue specimens using MasSpec Pen.

This analysis includes 95 lung cells (48 cancerous cells and 47 normal cells), 57 ovarian samples, 45 breast samples, and 56 thyroid samples. The samples were studied for histopathology. Only samples with predominant cell composition and proper diagnosis were used for the built molecular database.For further characterization of the study, they used lesson method to build classification model using the histological validated mass spectral databases.The accuracy of the process was around 96.3\%. MasSpec Pen was also successfully tested in the murine model of human breast cancer during surgery. The biggest success of this MasSpecPen is it could ensure removal of the last remnant of cancerous tissue while in surgery.

\section{How the MasSpec Pen works}

Our living cells (both noncancerous and cancerous cells) produce certain substances called metabolites, which are actually helping us in feeling energetic, growing, removing toxins and in reproduction. Each of the cancerous cells produces the certain type of biomarkers that actually acts as fingerprints. Cancerous metabolites are different from normal cell metabolites as because cancerous cells have deregulated metabolism. MasSpec Pen would analyze these biomarkers in the molecular label without causing any cellular or tissue damage. Using statistical classifier software biomarkers can instantaneously analyze. When MasSpec Pen analysis complete within 10 seconds, a mass spectrum appears within computer screen and gives labeled information as "Normal" or "Cancer" cells. For lung, cancer subtype might appear. It requires 
simple skills to operate, like holding the Pen probe against patient tissue, activating the automated analysis using the foot pedal and waiting for few second for results. After proper calibration of the instrument, the pen could release the drop of water $(10 \mu \mathrm{L}) 0 \mathrm{n}$ to the tissue and tissue molecules migrates into the water. Then the device carries mixed molecular water along by vacuum force generated by inert gas like nitrogen to the mass spectrometer. Furthermore, in mass spectrometer thousands of molecules detect as molecular fingerprints [12] (Figure 2).

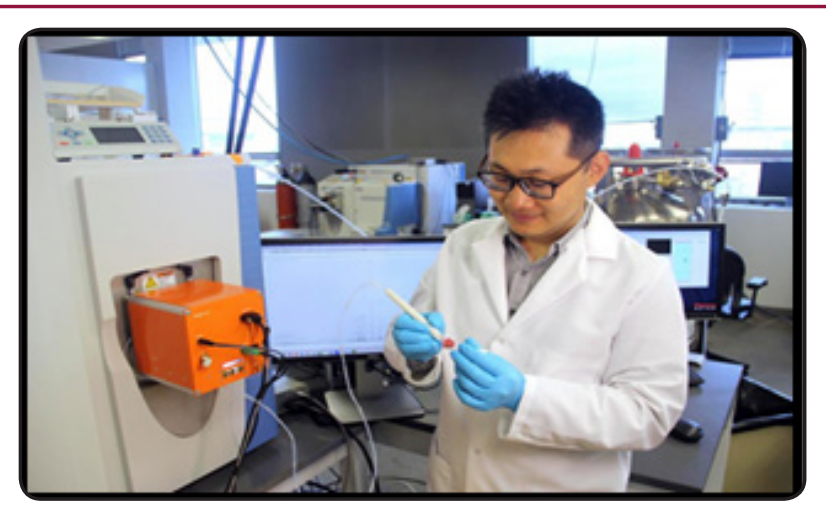

Figure 2 : J. Zhang with MasSpec Pen in University of Texas at Austin.

\section{Media headlines about MasSpec Pen}

1. CNBC news on $7^{\text {th }}$ September 2017 published news titled "scientist invent pen that identifies cancer in 10 seconds" [13].

2. BBC news outline the invention as "Pen' identifies cancer in 10 seconds" [14].

3. The verge had given hidings as "This cancer-detecting pen could one day help surgeon's better remove tumor" [15].

4. The Quarts published this invention as "Scientists invented a pen that can screen for cancer in seconds rather than weeks." [16].

5. The Sun bulletin gives a headline as "Pen-pointing Cancer: New handheld 'pen' allows surgeons to detect cancer in 10 Seconds - making sure they remove all trace of disease" [17].

6. On $6^{\text {th }}$ September 2017, New Scientist blog gives it a splendid headline as "Cancer pen could tell surgeons where they should cut in seconds" [18].

7. Chicago Tribune categorized the invention as "Cancer pen could help surgeons detect tumors in seconds" [19,20].

\section{Conclusion}

MasSpec Pen has several advantages over frozen section analysis in cancer diagnosis. In the frozen section, method during surgery, affected tissue would freezes and diagnosis may take not less than 20 minutes. However, using MasSpec Pen the job done within 10 seconds without giving much more pain to a patient. It can easily analyze the molecular fingerprints of cells, informing the surgeon whether the tissue is healthy or cancerous by which surgeon could easily remove the last verse of cancerous cells from the body. This technique helps in quicker, safer and precious surgery. Nevertheless, more work needs to be done to decrease mass spectrometer size and still an interpretation of samples needs more skills and knowledge.

\section{References}

1. J Zhang, J Rector, JQ Lin, JH Young, M Sans, et al. (2017) Nondestructive tissue analysis for ex vivo and in vivocancer diagnosis using a handheld mass spectrometry system. Sci Transl Med 9(406): eaan3968.

2. TA Buchholz, MR Somerfield, JJ Griggs, S El Eid, MEH Hammond, et al. (2014) Margins for breast-conserving surgery with whole-breast irradiation in stage I and II invasive breast cancer: American society of clinical oncology endorsement of the society of surgical oncology/ American society for radiation oncology consensus guideline. J Clin Oncol 32(14): 1502-1506.

3. SJ Maygarden, FC Detterbeck, WK Funkhouser (2004) Bronchial margins in lung cancer resection specimens: Utility of frozen section and gross evaluation. Mod Pathol 17(9): 1080-1086.

4. G Massard, C Doddoli, B Gasser, X Ducrocq, R Kessler, et al. (2000) Prognostic implications of a positive bronchial resection margin. Eur J Cardiothorac Surg 17(5): 557-565.

5. AM Nick, RL Coleman, PT Ramirez, AK Sood (2015) A framework for a personalized surgical approach to ovarian cancer. Nat Rev Clin Oncol 12(4): 239-245.

6. SS Han, JY Jang, SW Kim, WH Kim, KU Lee, et al. (2006) Analysis of longterm survivors after surgical resection for pancreatic cancer. Pancreas 32(3): 271-275.

7. K Chughtai, RMA Heeren (2010) Mass spectrometric imaging for biomedical tissue analysis. Chem Rev 110(5): 3237-3277.

8. CC Hsu, PC Dorrestein (2015) Visualizing life with ambient mass spectrometry. Curr Opin Biotechnol 31: 24-34.

9. CP Wu, AL Dill, LS Eberlin, RG Cooks, DR Ifa (2013) Mass spectrometry imaging underambient conditions. Mass Spectrom Rev 32(3): 218-243.

10. JL Norris, RM Caprioli (2013) Analysis of tissue specimens by matrixassisted laserdesorption/ionization imaging mass spectrometry in biological and clinical research. Chem Rev 113(4): 2309-2342.

11. DR Ifa, LS Eberlin (2016) Ambient ionization mass spectrometry for cancer diagnosis and surgical margin evaluation. Clin Chem 62(1): 111123.

12. LS Eberlin, I Norton, D Orringer, IF Dunn, XH Liu, et al. (2013) Ambient mass spectrometry for the intraoperative molecular diagnosis of human brain tumors. Proc Natl Acad Sci 110: 1611-1616.

13. Scientists (2017) The MasSpec Pen rapidly and accurately detects cancer in humans during surgery, Texas, Austin.

14. Scientists invent a pen that can detect cancer in 10 seconds (2017) Modern Medicine, USA.

15. Pen identifies in 10 seconds (2017) Health, USA.

16. The cancer detecting pen (2017) Health, USA.

17. Scientists invented a pen (2017) To C Or Not To C, Austin.

18. Pen pointing cancer (2017) A REVOLUTIONARY new handheld "pen" allows surgeons to test for cancer in seconds, Austin.

19. Can pen tell surgeons where they could cut (2017) Daily news, Austin.

20. Pen could help surgeons (2017) Chicago Tribune, Austin. 


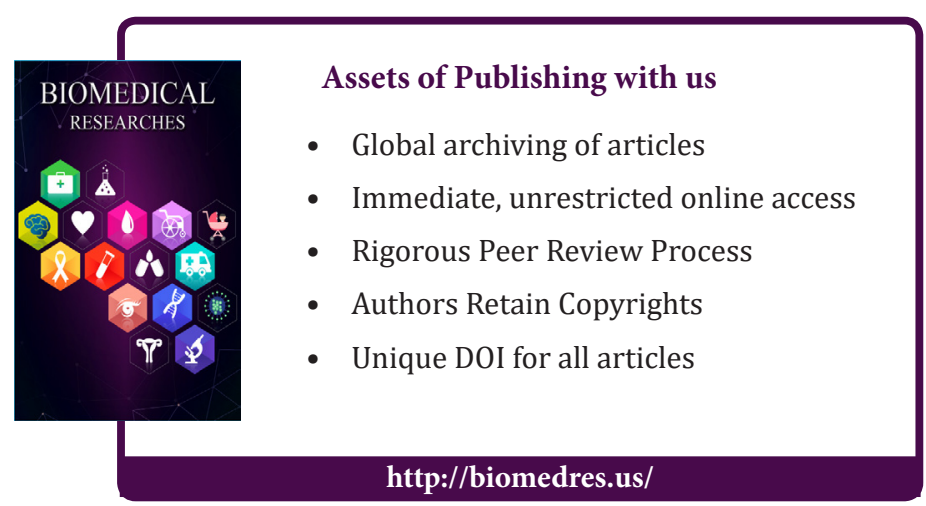

